International Journal of Ophthalmology \& Eye Science (IJOES)

ISSN 2332-290X

\title{
Low Vision Rehabilitation in the Glaucomas
}

McAllister B*, Rebecca Kammer

Diplomate, Low Vision, Western University of Health Sciences, College of Optometry, USA.

\section{*Corresponding Author:}

Bennett McAllister OD, FAAO,

Diplomate, Low Vision, Assistant Professor, Western University of

Health Sciences, College of Optometry, USA.

E-mail: bmcallister@westernu.edu

Received: August 13, 2014

Accepted: January 29, 2015

Published: February 04, 2015

Citation: McAllister B (2015) Low Vision Rehabilitation in the Glaucomas. Int J Ophthalmol Eye Res. S1:002, 5-9.

doi: http://dx.doi.org/10.19070/2332-290X-SI01002

Copyright: McAllister $\mathbf{B}^{\circ}$ 2015. This is an open-access article distributed under the terms of the Creative Commons Attribution License, which permits unrestricted use, distribution and reproduction in any medium, provided the original author and source are credited.

\section{Introduction to Low Vision Rehabilitation}

As seen elsewhere in this edition, the medical and surgical treatment of the glaucomas has advanced remarkably over recent years with enhanced diagnostic tools, greater variety of drug options and refined surgical procedures. Low vision rehabilitation has also seen advances with technology of adaptive devices, accessibility features on personal electronics and a synthesized rehabilitative model that incorporates aspects of the medical model of rehabilitation with the education/vocational system. This new model emphasizes identifying and ameliorating functional dependencies to improve patient quality of life (QOL) $[1,2]$.

Vision loss from the glaucomas is qualitatively different from other common etiologies that cause low vision in the developed countries. Age related macular degeneration (ARMD), the leading cause of reduced vision in seniors, whether dry (dARMD) or exudative (wARMD) primarily causes deficits in visual function impacting the central field while leaving peripheral visual function largely intact. Diabetic retinopathy (DR), the primary cause of vision loss in working age adults, negatively affects visual function in the central visual field through diabetic macular edema (DME) and peripherally through proliferative changes and pan-retinal photocoagulation (PRP) treatment. The glaucomas decrease visual abilities through a spectrum of central, paracentral and peripheral manifestations in what can be asymmetric and inconsistent patterns.

\section{Definitions}

Low Vision Rehabilitation practitioners have expanded the concept of glaucoma treatment beyond the narrow, medical and condition centered view to encompass treatment of functional seque- lae that impact the quality of life of individuals with glaucoma. The World Health Organization (WHO) has two definitions for low vision:

1. Low vision is visual acuity less than $6 / 18$ and equal to or better than $3 / 60$ in the better eye with best correction.

2. A person with low vision is one who has impairment of visual functioning even after treatment and/or standard refractive correction, and has a visual acuity of less than 6/18 to light perception, or a visual field less than 10 degrees from the point of fixation, but who uses, or is potentially able to use, vision for the planning and/or execution of a task for which vision is essential.

These two definitions, being based on visual abilities, do not address how the loss of function affects the individual in their activities of daily living (ADL) or quality of life (QOL). To address this gap, it is proposed that a third definition for low vision be added:

3. Low vision is a loss of visual function (i.e. Visual acuity, visual fields, and/or contrast sensitivity) caused by an organic or non-organic mechanism resulting in loss of functional ability and quality of life.

With the addition of ADL and QOL measures, the treatment of vision loss secondary to glaucoma becomes a natural extension to the current medical and surgical treatments. This is not an insignificant concept, especially to the many patients who are told nothing more can be done in the treatment of their glaucoma when what the doctor means is nothing more can be done medically or surgically to arrest, retard or cure the disease process [3]. Certainly we owe the patient a comprehensive treatment program beyond our own specialty. This can entail referrals and shared management to the improvement of our patients' ADL and QOL.

Incorporating the aspects of ADL and QOL, low vision in this paradigm can be viewed as a three part framework given the addition of the third definition of low vision above:

1. Cause: Visual abilities and their measures

2. Effect: How a person functions in their ADL given their visual abilities

3. Impact. How visual ability loss impacts QOL and how to ameliorate that impact

Cause refers to the loss of visual ability in the three main ability arenas of visual acuity, visual fields and contrast sensitivity function. Moreover, Cause looks to how such ability losses operate to interfere with optimal visual system performance. 
Effect in our parlance, deals with the level of functional visual ability and vision related performance independence given the associated Cause. In vision exams of the fully sighted, this is typically evaluated with standard health history questioning such as chief complaint (CC), review of systems (ROS) and past, family and social history (PFSH). Such questioning is frequently insufficient, however, when dealing with compounding issues in people with vision loss. Therefore, it is important to explore further with directed symptom surveys of functional independence measures (FIM) and observations of patient task performance.

$\underline{\text { Cause }}$ and $\underline{\text { Effect }}$, while important in traditional treatments of the glaucomas, together cannot reveal the extent of Impact the vision loss has on the quality of life of the individual patient. For this, we turn to patient reported, subjective life status rubrics and quality of life surveys. A number of validated vision related quality of life (VRQOL), health related quality of life (HRQOL) and more general QOL surveys exist to identify needs and direct appropriate referrals, education and resource allocation.

\section{Cause}

While there are a number of measureable attributes of visual ability commonly assessed in comprehensive exams, we find three whose dysfunction cause the greatest difficulty regarding patient complaints in reading, driving, facial recognition, mobility and fall risk in patients with glaucoma. These are:

1.visual acuity (VA),

2.visual fields $(\mathrm{VF})$ and

3.contrast sensitivity function (CSF).

\section{Cause - Visual Acuity}

Visual acuity can become problematic to quantify with standard office charts that were designed for the fully sighted patient when they are used with patients who have a glaucoma. Invalid measures, low levels of significance and poor outcome confidence in the resultant VA are consequences of acuities taken under the design assumptions of many common office VA charts. Of the four types of visual acuity, detection acuity, resolution acuity, Vernier acuity and recognition acuity, we find the most information from the later. It is also, the primary mover in getting people to present for a vision exam. Loss of form vision leads to poor reading, poor facial recognition and difficulties driving. Psychometric standards in measuring recognition acuity were strongly established by Bailey and Lovie in the late 1970s and refined in the early treatment of diabetic retinopathy (ETDRS) charts of the early 1980s [4]. Both utilize the concept of test task equivalence to yield high levels of confidence in the validity and repeatability of acuity measurements, allowing an accurate and consistent following of visual performance over time and tracking of treatment efficacy and disease progression.

Visual acuity taken at distance (20 feet or 6 meters or 4 meters) has been well established with the ETDRS charts, but near visual acuity and reading ability need further work and standardization.

There are four common systems of recording near visual acuity, 1. Jaeger $(\mathrm{J})$,

2. Point (n),

3. Reduced Snellen (RS) and

\section{M-Unit}

Only one, the M-Unit system, yields usable information for test distance and target size. In the field of low vision, flexibility in working distance and letter size is important in measurement and critical in treatment. Therefore, only M-units should be utilized.

\section{Cause - Reading}

Near visual acuity and reading ability are frequently conflated inappropriately when a reading task is assigned an acuity fraction. It becomes a recurring source of frustration for doctors and patients when near acuity measures taken in the office do not correspond to patient ability in activities of daily living [5]. Reading for information is a complex task of which near visual acuity is only one portion. Therefore, reading requires a more comprehensive description than near acuity alone [6]. A constellation of three factors has been established using the MN Read cards system which consists of,

\section{Reading Acuity,}

2. Maximum Reading Speed and

3. Critical Print Size.

Reading Acuity is the smallest resolvable word print size and is approximate to single digit near visual acuity. The top rate at which a patient can read contextual words for information is the Maximum Reading Speed while Critical Print Size is the smallest font the patient can read at the Maximum Reading Speed. Combining these three factors gives the doctor a more thorough understanding of their patient's reading ability and demonstrates to the patient some of the complexity involved in dealing with their functional vision deficit.

\section{Cause - Visual Field}

Most glaucoma patients receive many visual field tests through the course of their disease, but the type of visual field is typically chosen for tracking the progression of the neural pattern loss and not necessarily to track reading ability or Orientation \& Mobility (O\&M). This is, perhaps, unavoidable given the reimbursement schedules and need to follow standards of care in treating the underlying glaucoma disease process. In treating the person, however, the low vision rehabilitation specialist needs different visual field information to properly manage the patient's ADL and maximize their QOL.

Reading requires not just acuity, but context supplied by the field surround. Deruaz [7] contended that two conditions are required for good reading

\section{1. detail discrimination and}

\section{Global viewing.}

In languages that progress left to right, that field must precede the point of fixation to the right by about seven letters and trail to the left by three. Field defects to the right of fixation, therefore, are significantly more problematic for reading response than the equivalent defect to the left of fixation. Markowitz [8] held that three parameters of macular function should be assessed for reading ability

1.scotoma characteristics, 
2.preferred retinal loci (PRL) and

3.oculomotor control.

Unfortunately, the instrumentation required, microperimeters and scanning LASER ophthalmoscopes (SLO) are cost and time prohibitive for most low vision rehabilitation practitioners. The California Central Visual Field Test (CCVFT) was developed by Drs. Fletcher, Cole and Kammer to address this issue and provides significant information regarding the functional central visual field. While much of the reading work was done with patients who had macular degeneration, the concepts are generalizable for reading function [9].

\section{Cause-Contrast Sensitivity Function}

Contrast sensitivity function (CSF) is the third part of the visual ability triadmost commonly measured in a low vision rehabilitation evaluation. "Contrast" is the brightness difference between the highest and lowest brightness levels of a particular target. The ability to detect the minimum difference in contrast for a particular image size is designated "Contrast sensitivity" and the plot of Contrast Sensitivity to object size is termed "Contrast Sensitivity Function".A graph of contrast sensitivity function designates large objects to the left and small ones to the right. Most acuity charts measure only high contrast targets with decreasing size. Therefore, threshold visual acuity is a single point on the CSF plot to the far right. While no additive prescriptive value is found with CSF measures beyond that from acuity charts when using high contrast materials, ADL such as walking, cooking and grooming often consist of visual information of less than optimal contrast as well as various sizes. These ADL tasks can prove problematic for the patient beyond what one would expect given their visual acuity. In particular, mobility speed and safety (fall risk) are correlated with CSF [10]. Since visual acuity can be a good predictor of higher spatial frequency defects, CSF should be evaluated more with attention given to loss of contrast in the lower spatial frequencies. Losses in the lower spatial frequencies, however, are not generally amenable to treatment with optical devices like magnifiers or telescopes. The attendant functional losses of low spatial frequency deficits can though be ameliorated, however, with the techniques and training offered by Occupational Therapists (OT) and Orientation \& Mobility (O\&M) specialists.

\section{Effect}

Effect can be encapsulated as the functional sequelae of Cause. It is the functional losses that stem from the Cause we endeavor to uncover in order to initiate our targeted low vision rehabilitation treatment. In an exam of a fully sighted person, it is found to be generally adequate to ask about Chief Complaint (CC), Review of Systems (ROS) and Past, Family and Social History (PFSH). For the patient with reduced visual abilities secondary to a glaucoma, though, these are frequently insufficient. Consequently, it is important to investigate in a more comprehensive and systematic manner. We do this with symptom surveys of independence/dependence and observations of ADL.

\section{Effect-Directed Symptom Survey}

Activities of daily living are impacted by vision loss but not always addressed by the vision specific questions common in a vision exam. In order to more completely evaluate the low vision patient's level of ability and independence, the Functional Independent Measures (FIM) process is utilized. Herein, a seven point scale rubric of Independence-Dependence is used to separately investigate thirteen tasks of ADL. These tasks are: cooking, cleaning, grooming, finances, self-care, ambulating home, ambulating out, reading sustained, reading spot, other near tasks, intermediate tasks, distance tasks and technology.

Independent: Another person is not required for the activity (NO HELPER).

- Complete Independence - All of the tasks described as making up the activity are typically performed safely without modification, assistive devices (other than standard glasses or contact lenses) and within a reasonable time frame.

- Modified Independence - Activity requires any one or more of the following: An assistive device beyond standard glasses or contact lenses, more than reasonable time, or there are safety (risk) considerations.

Dependent: Another person is required for either supervision or physical assistance in order for the activity to be performed, or it is not performed (REQUIRES HELPER).

Modified Dependence: The subject expends 50\% or more of the needed effort to perform the task. The levels of assistance required are:

- Supervision or Setup - Subject requires no more help than standby, cueing or coaxing, without physical contact. Or, helper sets up needed items.

- Minimal Contact Assistance - With physical contact the subject requires no more help than touching, and subject expends $75 \%$ or more of the effort.

- Moderate Assistance - Subject requires more help than touching, or expends between $50 \%$ and $75 \%$ of the effort required to perform the task.

Complete Dependence: The subject expends less than $50 \%$ of the effort, maximal or total assistance is required, or the activity is not performed. The levels of assistance required are:

- Maximal Assistance: Subject expends between 25\% and $50 \%$ of effort for task

- Total Assistance: Subject expends less than 25\% of the effort

FIM scaling is a powerful tool to ascertain where a patient is having difficulty with an ADL, identify activities a patient has given up on or is avoiding, guide the rehabilitation team's planning, track treatment progress and modify care goals if necessary $[11,12]$. A directed symptom survey at the initiation of the low vision rehabilitation exam rationalizes the process for the doctor, directs the patient to possibilities they might not have considered and prepares them for thinking beyond just glasses and magnifiers.

\section{Impact}

The loss of vision abilities in visual acuity, visual field and contrast sensitivity function (Cause) bring about negative effectson ADL (Effect) which can lead to degradation in the person with glaucoma's quality of life (Impact). While Cause and Effect are appropriately addressed during the evaluation process of the physician and therapist, Impact may need to be attended to more broadly than the traditional low vision rehabilitation devices and 
techniques have addressed.

\section{Impact-Quality of Life}

There is no precise, accepted definition of quality of life, though the World Health Organization loosely defines QOL as, "an individual's perception of their position in life in the context of the culture and value systems in which they live and in relation to their goals, expectations, standards and concerns. It is a broad ranging concept affected in a complex way by the person's physical health, psychological state, personal beliefs, social relationships and their relationship to salient features of their environment."[13] This rather broad definition covers the various conceptualizations and instruments which generally tend to include both objective and subjective measures in a multi-dimensional framework that acknowledges individual, societal and economic values. They include evaluations of a person's physical, material, social and emotional well-being that present an overall frame of reference to the patient's QOL. Instruments and tools used to gauge QOL tend to be long and time consuming to administer, their primary drawback, but have been used to validate the efficacy of the low vision rehabilitation model at several levels.

Beyond overall QOL, the Center for Disease Control as parsed out those aspects of overall quality of life pertaining to health, or health related quality of life (HRQOL) [14]. This was done to better direct allocation of scarce resources and to improve public health decision making. Vision related quality of life (VRQOL) is, as its name suggests, an attempt to further develop tools to measure the impact of vision loss on QOL, though so far without widespread adoption [15].

Patient reported outcomes (PRO) are used by third party and government regulators to assess quality of care from the patient and societal viewpoint. Of the 121 vision related PRO reported by Khadka, McAlinden and Pesudovs in 2013, only 48 studies met the quality inclusion criteria of question unidimensionality and interval-level measurement [16]. Of those, only 6 were related to low vision rehabilitation with the Veterans Affairs Low-Vision Functioning Questionnaire considered the highest quality. This PRO was validated in the Veteran Affairs Low Vision Intervention Trial (LOVIT) which reported significant improvement in every facet of visual function outcomes for patients who received low vision rehabilitation treatment. Consisting of 48 questions, this instrument takes too long to administer to be used on every low vision rehabilitation patient, and, as with all current PRO, was limited by the fixed question format which did not allow for adaptive questions based on preceding answers. Still, the LOVIT study clearly showed improved QOL for patients with vision loss who received low vision rehabilitation.

\section{Impact - Depression}

Renaud and Bedard in 2013 reviewed the literature for linkages between depression and vision impairment [17]. As expected, their findings indicate that better quality of life is strongly related to less severe depressive symptoms. Eramudugoll, Wood and Anstey were able to show a significant association between objective indices of visual impairment such as VA, visual fields, contrast sensitivity, and functional vision loss with depressive and anxiety symptoms [18]. While this would seem to point to the need for training of vision professionals in the screening and referral of visually impaired (VI) patients for depression, there is no widespread program in place [17].

\section{Impact-Vision Enhancement}

The mainstay of low vision rehabilitation for decades has been vision enhancement. This can be achieved through optical devices such as high dioptric powered readers, hand held magnifiers, stand magnifiers and hand held telescopes. Traditionally, the devices were dispensed to patients with minimal instruction and training. Evidence that patient usage of and facility with optical devices increases with evaluation of ADL, lifestyle and cognitive dimensions as well as formalized training in device use has led to a change in the low vision rehabilitation paradigm to include rehabilitation therapists. A growing trend is for the low vision rehabilitation physician to work with a low vision trained occupational therapist (OT) to train with the devices and encourage lifestyle adaptations for the patient over the course of rehabilitation [19]. Low vision rehabilitation is not limited to optical devices. Nonoptical devices, traditionally lighting and glare control devices and techniques, have expanded dramatically with the micro-electronic revolution. Adaptive features are imbedded in every smart phone and tablet allowing orders of magnitude increase in accessibility of information and GPS orientation. In fact, the number of specialized apps for the visually impaired have increased substantially over the recent years and in an attempt to assist users, the Braille Institute developed an app to find, organize and sorteach app into categories [20].

\section{Impact - Vision Substitution}

Unfortunately, a patient undergoing glaucoma treatment may lose vision beyond the point where some or all of the functional independence measures are no longer positively impacted by vision enhancement devices, techniques or training. This is the time when the low vision rehabilitation team segues to the devices, techniques and training of vision substitution. Vision enhancement and vision substitution are not mutually exclusive, however. In some of the thirteen category of functional independence, the patient may perform satisfactorily with a form of vision enhancement but require vision substitution to maintain functional independence in another category.As technology provides ever more and better accessibility options, the traditional barriers hindering people with visually impairment (VI) from fully participating in activities available to the fully sighted are being pushed back and, in some cases, overcome.

It is not a stretch to imagine an autonomous (self-driving) car taking a low vision or blind adult to the airport where their GPS guides them to the proper gate for a business trip or a VI student traveling independently by bus through app instructions and interacting with their lesson plan by verbal commands and feedback or refreshable Braille driven by optical character recognition (OCR) software. Vision loss no longer has to mean functionality loss if the low vision practitioner employs the proper knowledge, tools, devices, training and referrals for their patient.

\section{Conclusion}

Low vision rehabilitation of the patient with vision loss from a glaucoma, optimally practiced, should involve an interprofessional approach with optometrists, ophthalmologists, occupational therapists, orientation and mobility specialists, technology specialists, social or rehabilitation workers and teachers of the visu- 
ally impaired. The paradigm of Cause, Effect and Impact expands and enhances the treatment spectrum for patients with glaucoma beyond the medical and surgical to embrace an emphasis on addressing functional dependencies in activities of daily living and improving quality of life.

\section{References}

[1]. Massof RW, Lidoff L (2001) Issues in Low Vision Rehabilitation: Service Delivery, Policy and Funding, AFB Press, New York.

[2]. Owsley C, McGwin G Jr, Lee PP, Wasserman N, Searcey K (2009) Characteristics of Low-Vision Rehabilitation Services in the United States. Archives of Ophthalmology 681-689.

[3]. JH Tranton (1994) Nothing More Can Be Done...A Fable for our Times. Ophthalmology Clinics of North America 7(2).

[4]. Bailey IL, Lovie-Kitchen JE (2013) Visual Acuity Testing. From the Laboratory to the Clinic. Vision Research. 90: 2-9.

[5]. J Lovie-Kitchen (2011) Reading with Low Vision: the Impact of Research on Clinical Management. Clinical Experimental Optometry 94(2): 121-32.

[6]. Falkenberg HK, Rubin GS, Bex PJ (2007) Acuity, Crowding, Reading and Fixation Stability. Vision Research 47(1): 126-35.

[7]. Deruaz A, Goldschmidt M, Whatham AR, Meroud C, Lorincz EN, et al. (2006) A Technique to Train New Oculomotor Behavior in Patients with Central Macular Scotomas During Reading Related Tasks Uning Scanning LASER Opththalmoscopy:Immediate Functional Benefits and Gains Retention. BMC Ophthalmology 23(6).

[8]. Markowitz S, Reyes S (2013) Preferred Retinal Loci and Macular Scotoma Characteristics in Patients with Age-Related Macular Degeneration. Canadian Journal of Ophthalmology 48(5).

[9]. Kammer R, Poulter C, Clark J (2009) The Effect of Altered Stimulus Luminance on the Size of Scotomas Mapped with the Fletcher Central Visual Field Test. Invest Ophthalmol Vis Sci 50: 4738.

[10]. Pelli DG, Bex P (2013) Testing Vision: From Laboratory Psychophysical Tests to Clinical Evaluation-Measuring Contrast Sensitivity. Vision Research 90: 10-14.

[11]. Granger CV, Deutsch A, Linn RT (1998) Rasch Analysis of the Functional Independence Measure (FIM) Mastery Test. Archives Physical Med Rehabilitation 79(1): 52-57.
[12]. Young Y, Fan MY, Hebel JR, Boult C (2009) Concurrent Validity of Administering the Functional Independence Measure (FIM) Instrument by Interview. American Journal Physical Med Rehabilitation 88(9): 766-770.

[13]. World Health Organization (1995) World Health Organization Quality of Life Assessment (WHOQOL): Position Paper. Soc Sci Med 41(10): 14031409.

[14]. Center for Disease Control. Position Paper Health Related Quality of Life. www.cdc.gov/hrqol/concept.htm.

[15]. de Boer MR, Moll AC, de Vet HC, Terwee CB, Volker-Dieben HJ, et al. (2004) Psychometric Properties of Vision-Related Qouality of Life Questionnaires: a Systematic Review. Ophthalmic Physician Opt 24(4): 257-273.

[16]. Khadka J, McAlinden C, Pesudovs K (2013) Quality Assessment of Ophthalmic Questionnaires: Review and Recommendations. Optom Vis Sci 90(8): 720-744.

[17]. Renaud J, Bedard E (2013) Depression in the Elderly with Visual Impairment and its Association with Quality of Life. Clin Interv Aging 8: 931-943.

[18]. Eramudugolla R, Wood J, Anstery KJ (2013) Co-Morbidity of Depression and Anziety in Common Age-Related Eye Diseases. Frontiers in Aging Neuroscience 5: 56 .

[19]. Liu CJ, Brost MA, Horton VE, Kenyon SB, Mears KE (2013) Occupational Therapy Interventions to Improve Performance of Daily Activities at Home for Older Adults with Low Vision: A Systematic Review. Am J Occup Ther 67(3): 279-87.

[20]. Braille Institute.Visually Impaired Apps (VIA). https://itunes.apple.com/in/ app/via-by-braille-institute/id528499232? $\mathrm{mt}=8$

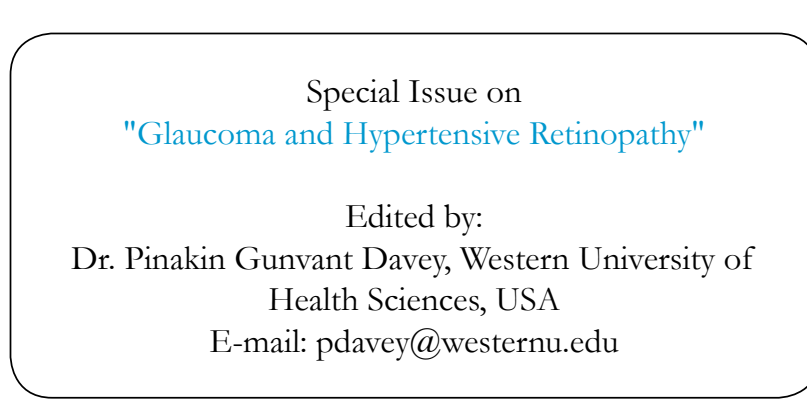

